\section{Biliary ascariasis as etiology of recurrent abdominal pain}

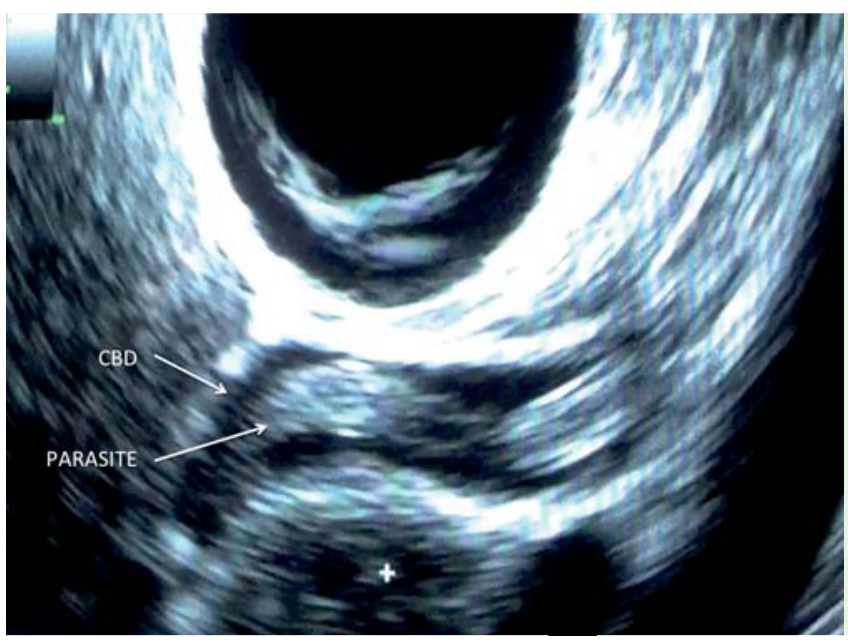
graphic view of Ascaris lumbricoides parasite inside the common bile duct (CBD).

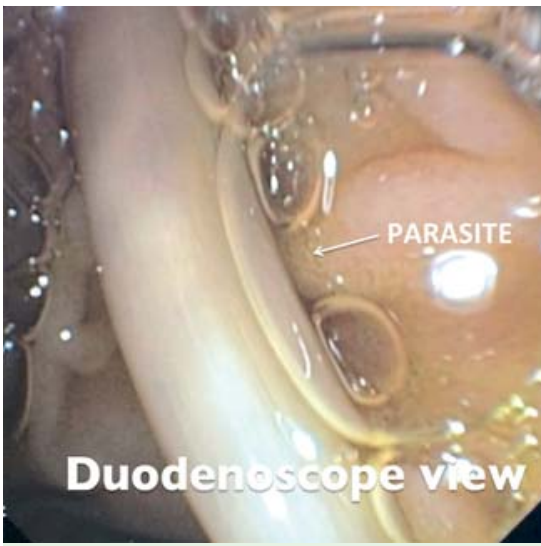

Fig. 2 Endoscopic view of Ascaris lumbricoides exiting from the major duodenal papilla.

A 37-year-old woman had a history of cholelithiasis and choledocholithiasis in 2007, and cholecystectomy and endoscopic retrograde cholangiopancreatography (ERCP) with sphincterotomy had been performed.

She was admitted to our unit with a 10-day history of upper abdominal pain that radiated into the back. She reported no weight loss, no fever, and no change in bowel habit. On questioning, the patient reported that she had travelled to Peru 6 months previously, and had probably ingested contaminated water. Laboratory tests showed minimal elevation of serum amylase, and normal findings for lipase, hepatic function, complete blood count, electrolyte levels, and blood chemistry. Abdominal ultrasound revealed hepatic steatosis.

Mild pain persisted despite treatment with antispasmodics. After 5 days, upper gastrointestinal endoscopy was per-

Diagnosis and removal of Ascaris lumbricoides parasite from the common bile duct.

formed and showed antral chronic gastritis. Endosonography showed normal large blood vessels. The uncinate process, head, isthmus, body, and tail of the pancreas showed normal appearances, as did the size and shape of the papilla and pancreatic duct. The common bile duct was dilated to $9 \mathrm{~mm}$, and a tubular entity about $4 \mathrm{~mm}$ in diameter was extending through it, from the duodenal lumen to the intrahepatic bile duct. Tubular structures that moved independently were observed ( Fig.1). Because the endoscopic appearance was suspicious for parasitic infestation, we exchanged the echoendoscope for a duodenoscope, confirmed the diagnosis ( $\bullet$ Fig. 2 ), and extracted the specimen completely using a Talon grasping device (US Endoscopy, Mentor, Ohio, USA), observing a parasite of $20 \mathrm{~cm}$ in length ( Video 1 ).

Microbiological study confirmed Ascaris lumbricoides. After extraction of the parasite, the patient reported no abdomi-
Fig. 1 Endosono-

nal pain. A single dose of albendazole (400 mg) was administered [1] and the patient was discharged.

Ascaris lumbricoides is an intestinal nematode (roundworm), producing a globally distributed helminthic infection. The majority of infections occur in the developing countries of Asia and Latin America [2]. The symptoms and complications of infection can be pulmonary and hypersensitivity manifestations [3], intestinal symptoms and obstruction [4], and hepatobiliary and pancreatic [5].

Endoscopy_UCTN_Code_CCL_1AF_2AF_3AZ

Competing interests: None

\section{Rodrigo Mansilla-Vivar ${ }^{1}$, Elizabeth Sainza Caballero ${ }^{2}$, Cristina Saldaña Dueñas $^{2}$, Laura Casanova Ortiz ${ }^{2}$, Ignacio Fernandez-Urien², Juan J. Vila Costas ${ }^{2}$}

${ }^{1}$ Department of Gastroenterology, Pontificia Universidad Católica, Santiago de Chile, Chile

2 Department of Gastroenterology, Complejo Hospitalario of Pamplona, Spain

\section{References}

1 Norhayati M, Oothuman P, Azizi O et al. Efficacy of single dose albendazole on the prevalence and intensity of infection of soil-transmitted helminths in Orang Asli children in Malaysia. Southeast Asian J Trop Med Public Health 1997; 28: $563-569$
Bibliography

DOI http://dx.doi.org/

10.1055/s-0042-107593

Endoscopy 2016; 48: E196

(c) Georg Thieme Verlag KC

ISSN 0013-726X

\section{Corresponding author}

\section{Rodrigo Mansilla-Vivar, MD}

Pontificia Universidad Católica de Chile

Departamento de Gastroenterología

Marcoleta 367

Santiago de Chile 8330024

Chile

Fax: +56-2-3543820

rmansillavivar@gmail.com
Stuttgart · New York 